\title{
Towards achieving semantic interoperability of clinical study data with FHIR
}

\author{
Hugo Leroux* (D), Alejandro Metke-Jimenez and Michael J. Lawley
}

\begin{abstract}
Background: Observational clinical studies play a pivotal role in advancing medical knowledge and patient healthcare. To lessen the prohibitive costs of conducting these studies and support evidence-based medicine, results emanating from these studies need to be shared and compared to one another. Current approaches for clinical study management have limitations that prohibit the effective sharing of clinical research data.

Methods: The objective of this paper is to present a proposal for a clinical study architecture to not only facilitate the communication of clinical study data but also its context so that the data that is being communicated can be unambiguously understood at the receiving end. Our approach is two-fold. First we outline our methodology to map clinical data from Clinical Data Interchange Standards Consortium Operational Data Model (ODM) to the Fast Healthcare Interoperable Resource (FHIR) and outline the strengths and weaknesses of this approach. Next, we propose two FHIR-based models, to capture the metadata and data from the clinical study, that not only facilitate the syntactic but also semantic interoperability of clinical study data.

Conclusions: This work shows that our proposed FHIR resources provide a good fit to semantically enrich the ODM data. By exploiting the rich information model in FHIR, we can organise clinical data in a manner that preserves its organisation but captures its context. Our implementations demonstrate that FHIR can natively manage clinical data. Furthermore, by providing links at several levels, it improves the traversal and querying of the data. The intended benefits of this approach is more efficient and effective data exchange that ultimately will allow clinicians to switch their focus back to decision-making and evidence-based medicines.
\end{abstract}

Keywords: FHIR, CDISC ODM, Interoperability, Clinical research data, Longitudinal clinical study

\section{Background}

Clinical research plays a vital role in advancing medical knowledge and improving clinical outcome. It is becoming increasingly clear that results from clinical studies need to be shared and compared to one another in order to support efficient evidence-based medicine [1] and reduce the costs of conducting these studies. By the same token, Hsu et al. [2] argue that to fulfil the goals of precision medicine requires the mining and aggregation of clinical data from multiple sources and entails novel approaches to obtaining contextual observations. Hume et al. [3] state that: "clinical research can no longer be considered an isolated venture and is increasingly conducted in network

${ }^{*}$ Correspondence: hugo.leroux@csiro.au

The Australian E-Health Research Centre, CSIRO Health and Biosecurity, Level 5, Health Sciences Building 901/16, Royal Brisbane and Women's Hospital, Herston 4029, Queensland, Australia structures where seamless data exchange is critical to operational efficiency and effectiveness". The challenge when comparing results from different data sets is to ensure that we are comparing corresponding data sets.

The Operational Data Model (ODM) [4] is an $\mathrm{XML}^{1}$ based standard from the Clinical Data Interchange Standards Consortium (CDISC) that was originally developed to facilitate the exchange, archival and audit trail requirements of clinical information but whose use has been extended to cover cases not initially anticipated [3], such as integrating health records within clinical research systems. The Federal Drug Administration has mandated the use of the CDISC standards for the electronic capture and reporting of clinical study data [5]. ODM is particularly well-suited for a data capture context [3, 6-11]. It is a mature data interchange standard that has proven useful for exchanging both document and message formats [3]. 
Its strength is in its relative simplicity, adaptability through the use of extensions [3] and in its ability to support the creation of a broad range of customisable Clinical Report Forms (CRFs) [3, 12].

ODM, however, lacks a rich-enough information model to capture the innate contextual information of the clinical study data $[7,13]$. Its relative simplicity, has impacted on its ability to advance all aspects of interoperability, limiting its support for data mapping, data types, terminology and semantic representation [3]. In spite of its efficacy as a data interchange, ODM has some shortcomings in the mapping of semantically identical data elements due to lack of support for semantics associated with the data elements $[3,11]$.

ODM can be considered to represent syntactic interoperability (as defined by [14]) of clinical data as it provides a vehicle for clinical data to be shared using an XMLbased model. However, our aim is to achieve semantic interoperability. Semantic interoperability is the ability, for health information systems, to exchange information and automatically interpret the information exchanged meaningfully and accurately in order to produce useful results as defined by the end users of both systems $[14,15]$. Extensions to ODM, such as the Clinical Data Acquisition Standards Harmonization (CDASH) [16] and the Biomedical Research Integrated Domain Group (BRIDG) [17] provide a reference model, although as stated by [18]: "studies that use CDASH CRFs achieve semantic alignment through a shared data standard, rather than through specific semantics". Furthermore, there is no requirement for the CDASH model to be used within ODM [7]. Moreover, uptake of CDASH and BRIDG to provide data semantics has been limited [3]. As a result, ODM is ill-suited for advancing the semantic interoperability solution that is required to achieve cross-study exploration of the clinical studies as there is the potential for the data to be interpreted in a way that was not originally intended by the study initiators.

The ability to achieve cross-study analysis also necessitates clinical studies to adopt a more streamlined data structure [7]. However, the monolithic nature of the ODM data model favours a one-dimensional traversal of the clinical data along its hierarchy of Study-Subject-StudyEvent-Form-ItemGroup-Item. More effective exploration and querying of the clinical data, especially when dealing with longitudinal studies, requires more direct access to the data, particularly at the Study Event, Subject and Item levels $[6,7,19]$.

The Fast Healthcare Interoperable Resources [20] (FHIR) framework, a HL7 ${ }^{2}$ standard that has been swiftly adopted by the health-care community [21-23], looks the likely candidate for overcoming this challenge. It is geared towards communication of clinical data using HL7 messaging protocols but is also supported by a rich information model to achieve semantic interoperability of clinical data. This makes FHIR the natural match to complement the ODM standard [8] as ODM shares several design principles, such as making use of extensions for edge cases and human readability, with FHIR [3]. Furthermore, FHIR has the potential to incorporate existing electronic health record (EHR) data to augment the findings of retrospective observational studies. As intimated by Kubick [24], "FHIR can make it possible to reach inside of EHRs not just to capture data, but to monitor protocol progress, provide safety alerts, and allow much greater visibility into trial conduct and can lead to dramatic improvements in study efficiency and drug safety".

This research builds upon the approach [8] to integrate clinical data extracted in CDISC ODM format into several FHIR resources with a view to achieving semantic interoperability of clinical study data. In the next section, we outline the approach taken to map the ODM-based data and metadata onto eight FHIR resources. In particular, we outline the suitability of the FHIR resources in supporting the ODM model and on all the assumptions made to reintroduce the contextual information to the data. We then critique this approach. Consequently, we propose the FHIR ClinicalstudyPlan resource to capture the clinical study metadata, including the potential to encode the study protocol as part of the model. This is followed by a description of the FHIR ClinicalstudyData resource that describes the clinical study data. Finally, it leads into a discussion on the design principles of the two proposed FHIR resources and on their suitability for representing clinical study data.

\section{Integrating ODM with FHIR}

This section outlines the approach described in [8] to integrate the ODM data model to a selection of eight FHIR $^{3}$ resources to capture both the data and metadata properties of the ODM data model. The CDISC ODM data model [4] consists of two main hierarchies: a Clinical Data and a Metadata hierarchy, as depicted in Fig. 1, that are referenced using the same object identifier (OID). These two parallel hierarchies ensure that the clinical study follows a predetermined structure of subject, event, form, item group and item. Figure 2 outlines the FHIR resources chosen to model the ODM data. The entities in red (CarePlan and Questionnaire) denote metadata concepts. The remaining entities, in blue, model the clinical data at various levels of the ODM hierarchy. Solid lines are used to denote the links between the entities.

The approach taken to map the ODM data into FHIR resources is a semi-automatic process. As the ODM data model does not natively provide any mechanism to capture the contextual information relating to the study, the data semantics needs to be re-introduced during this process. This can only be achieved if the person doing the 


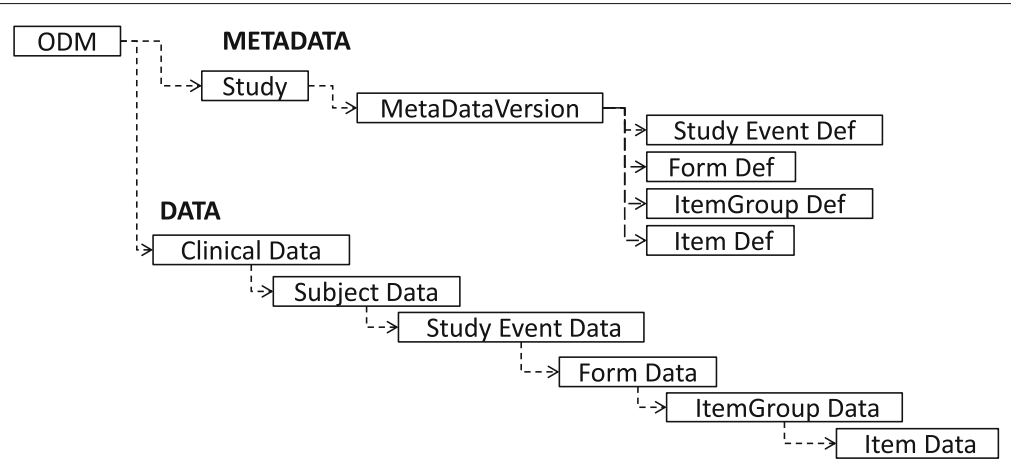

Fig. 1 The ODM data model. Illustrates the logical organisation of the ODM model into the data and metadata hierarchies

mapping has access to all the conceptual information defining the study. Figure 3 illustrates how the hierarchical ODM model has been mapped to the FHIR resources.

\section{Study}

A study defines static information about the structure of an individual study. We choose to model the Study component from ODM using the CarePlan resource because we want to model the activities planned for the patient during the study in the context of the study protocol. CarePlan provides a link to the study coordinator through the participant attribute and study protocol through the support attribute. Furthermore, the CarePlan resource offers a number of attributes, such as context, category and description that can provide additional context to the care plan.

\section{Subject}

While the Subject represents a critical element of the study, its role is quite subdued in ODM. In particular, the specification provides no functionality to record the subject's attributes such as gender or date of birth, recommending that these be modelled as clinical data within the forms. The logical mapping for the Subject in FHIR is the Patient resource. Relevant contextual information, such as the patient's gender, date of birth and care provider, can be encapsulated within the resource. The clinical data for each subject is contained within a ClinicalImpression resource that is linked to the Patient resource. The care plan is linked to this resource using the plan attribute. The Clinical Impression permits very pertinent information to be associated to the patient's data through the

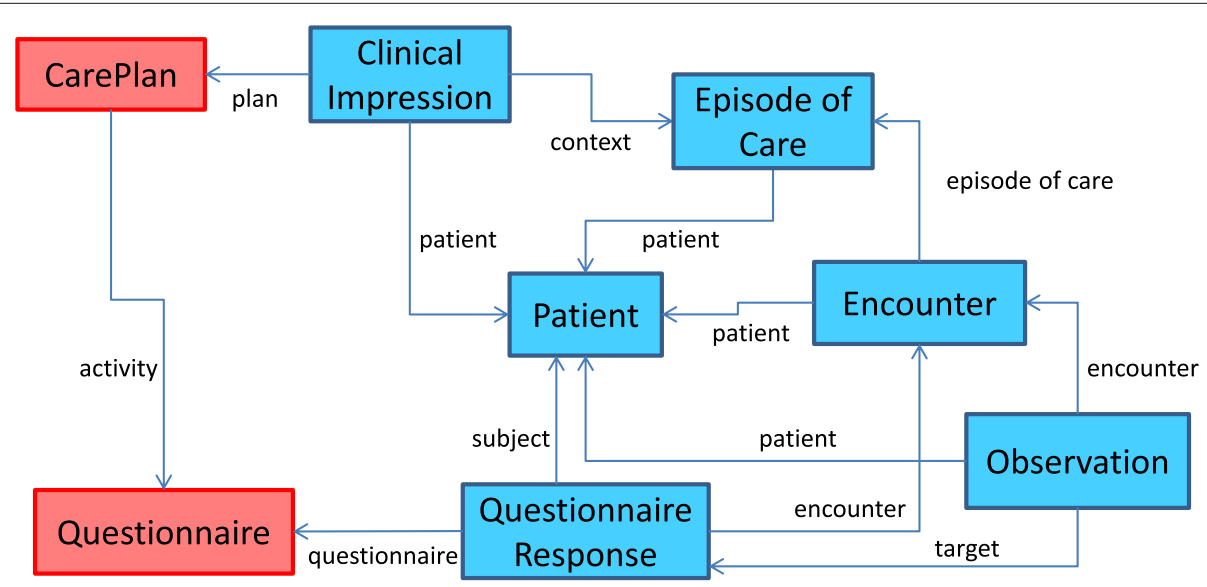

Fig. 2 The FHIR data model. Depicts the metadata (red) and data (blue) FHIR resources and their links that comprise the data model to transform the clinical data from ODM to FHIR. The CarePlan and Questionnaire resources are used to capture the metadata for the study. A Pat ient resource is used to represent the study participant while the clinical data for this participant is contained within a Clinical Impression resource. The study events are captured within the Epi sodeof Care resource and the Encounter resource represents one atomic event. The QuestionnaireResponse resource captures the form responses and the Observation resource illustrates those responses that are analogous to a patient's observations. The QuestionnaireResponse resource is linked back to the Questionnaire resource 


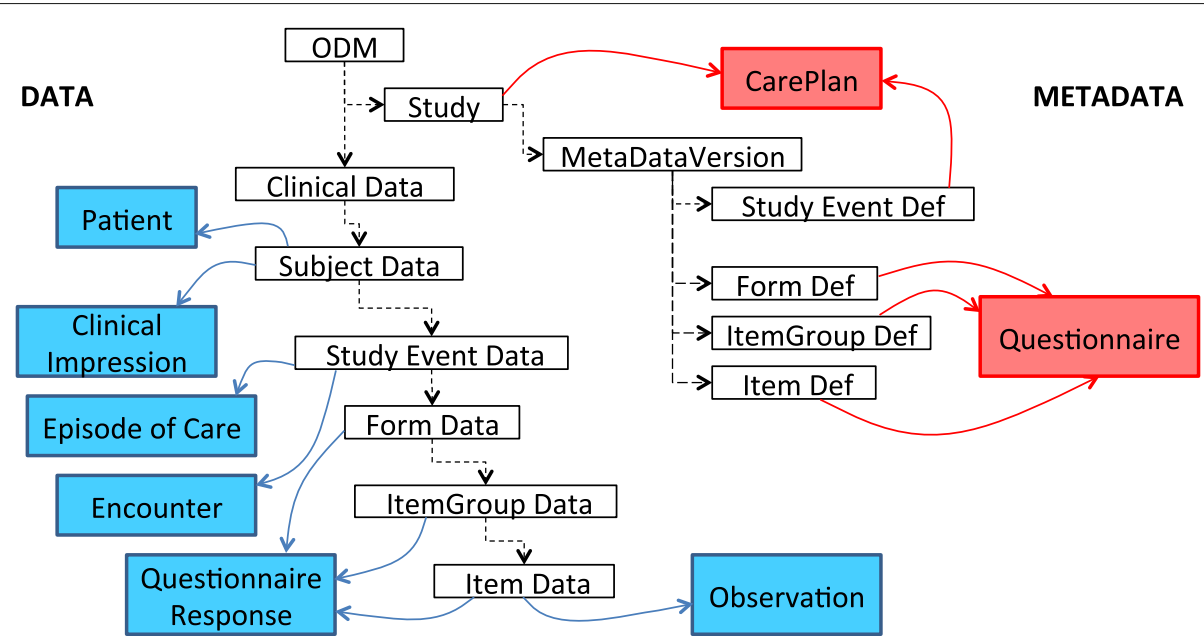

Fig. 3 Mapping the ODM data model to the FHIR resources. Illustrates how the CDISC ODM model (depicted by unshaded rectangles) is overlaid with the FHIR resources. The Met adata section, depicted on the right of the model with red rectangles to represent the FHIR resources, is mapped to the CarePlan resource at the Study and Study Event level, and to the Questionna ire resource to represent the form and its composition. The Data section is depicted on the left of the model with the FHIR resources depicted as blue rectangles. The Patient resource represents the study participant. The Clinical Impress ion resource captures the clinical data for this participant and they are both linked to the ODM model at the Subject Data level. As both the Epi sodeOfCare and Encounter resource correspond to study events, they are mapped at the StudyEventData level. The Questionna ireResponse resource captures the form responses and is linked to the form data and its composition. Finally, the Observation resource is used to capture those responses that are more analogous to a patient's observations

use of the trigger, investigations and summary attributes.

\section{Study event}

A study event comprises a StudyEventDef and a StudyEventData component that are referenced using a common OID. The StudyEventDef manages the set of forms to be completed at this phase of the study and represents an activity within the CarePlan resource. StudyEventDef entities define scheduled and unscheduled events and these are defined within the detail.scheduled attribute of the activity. The StudyEventData entity contains clinical data collected during a subject's visit. We chose the EpisodeofCare resource for this entity because it provides details about the group of activities and their purpose pertaining directly to a patient. A study event may result in many visits from a patient. Each individual visit is modelled as an Encounter and is linked to the episode of care through the episodeofCare attribute. The patient attribute links the resource to the study subject while the assessor attribute provides a link to the clinician conducting the clinical assessment.

\section{Form}

A form defines a collection of data items collected during the study and termed a case report form. A form comprises a FormDef and a FormData component that are referenced using a common OID. The form is linked to CarePlan through the activity. actionResulting attribute. The FormDef defines the form structure and its questions. The logical mapping of forms in FHIR is the Questionnaire resource. This resource contains the typical attributes for questionnaires, such as an identifier, version, publisher and status, but can also be customised using the extension mechanism in FHIR. The FormData entity contains the clinical data associated with the form. The logical mapping for the FormData in FHIR is the QuestionnaireResponse resource. The benefits of using the QuestionnaireResponse resource are that the order of the responses is maintained and these can be linked and validated against the questions asked. Conversely, however, few mechanisms exist to standardise the generation of CRFs for clinical studies $[8,11]$. This limits the reuse of CRFs unchanged across protocols [11]. Furthermore, the tendency is to organise data items, relevant to a research protocol, into individual CRFs based on considerations other than logical grouping $[8,11]$ but one that befits the data capture process [8]. Owing to the strong coupling between the form design and the ODM model, until such a time that implementations of ODM allow for a clear demarcation between the form design and its display, we advise against modelling the CRF per se as a FHIR resource [7].

\section{Item group}

The ItemGroupDef and ItemGroupData entities constitute an item group referenced using a common OID. 
The ItemGroupDef entity defines the optional grouping of questions on a form. Groups are defined using the Questionnaire group attribute. The FHIR specification stipulates that a group attribute define either a question or a group but not both. The ItemGroupData contains the clinical data detailing the responses for the item group. FHIR organises these grouped responses within the QuestionnaireResponse.group attribute. Similar to forms, items are often grouped to match the data collection process and not necessarily because of their semantic similarity $[8,11]$.

\section{Item}

At the item level, the ItemDef and ItemData entities define each question and its subsequent response. The ItemDef entity defines the question asked during the study along with defining attributes such as the datatype, data size, measurement unit, permissible range and code list. The Questionnaire.group.question attribute is the most appropriate to define the ItemDef entity. The logical mapping for the ItemData entity is the QuestionnaireResponse.group.question attribute. The response to the question is then contained within the question.answer sub-attribute. This model works best in a lifestyle study scenario using questionnaires in the traditional question-answer mode. In the case of longitudinal clinical studies where the responses are analogous to a patient's observations during an episode of care, we believe the ItemData entity to be more appropriately represented using the Observation resource. Furthermore, as outlined in the FHIR specifications, data captured in questionnaires can be difficult to query after the fact. Individual items within a QuestionnaireResponse or an Observation are subsequently linked back to the Encounter in which they occur.

\section{Discussion}

An implementation of the mapping between ODM and FHIR is available at http://healthinet.it.csiro.au/ net/jbs/odmFhir. We have semantically enriched the original ODM data with relevant domain information from SNOMED CT ${ }^{4}$ and LOINC 5 . The implementation demonstrates that the FHIR resources provide a good fit to semantically enrich the extracted data from the CDISC ODM. In spite of its shortcomings in providing context to the clinical data, the CDISC ODM provides a sound hierarchical framework for capturing the clinical data. However, as outlined in [25], a mapping process invariably leads to the loss of pertinent information. On the metadata side, for example, a study is modelled as a CarePlan. The CarePlan resource, however, is not used in its intended manner in that it does not relate to a particular individual. Similarly, despite being chosen to capture the clinical data, the Clinical Impression resource has no capability to model the study hierarchy. As a result, it relies on several other FHIR resources, such as EpisodeofCare and Encounter, which are also not used as intended, to describe the hierarchy. As stated by Kubick [24], it is preferable to avoid data transformations, if possible, especially when this involves massaging the data to fit into different formats, as this opens up the possibility of introducing errors and reducing the data reliability. Another issue relates to discrepancies between the data types defined within the ODM and FHIR models. In addition to the type, ODM allows the permissible range of the resulting data and, in the case of decimal values, the length of the permissible value to be defined. The answer attribute within the QuestionnaireResponse resource has no such capability. The observation resource is the only one to allow such a definition.

The main challenge of the mapping process, however, relates to the FHIR specifications. Being an emerging and evolving standard, FHIR is in a great state of flux. As such, FHIR resources are constantly being updated between releases. The implications are that relationships described using one version of the FHIR specifications may no longer be available in a subsequent version. The Questionnaire and Clinicalimpression resources are two resources that have undergone several changes.

\section{Clinical study design using FHIR}

Kubick [24] advocates (i) for the adoption of FHIR for clinical research; (ii) for clinical data to be captured directly at the source; and, (iii) for data transformation to be avoided whenever possible. Similarly, Huser et al. [10] argue that the adoption of a single format for study protocols and study results decreases the development time required to import studies into the repository or to exchange data between systems. Besides, the FHIR model has the potential to manage clinical data in its own right [8]. Consequently, we propose the introduction of two new FHIR resources to capture the data and metadata from the clinical study. These resources have been integrated in a data model, as illustrated in Fig. 4, which corresponds to the mapping, in FHIR, for a typical research study. The ClinicalstudyPlan resource, outlined in Fig. 5, defines the study and provides an overview of the planned activities. The ClinicalstudyData resource, outlined in Fig. 6, describes the data captured as part of the study organised around the events and visits of the patient.

\section{ClinicalStudyPlan}

The ClinicalstudyPlan resource comprises several attributes to capture the fundamental concepts within the study. Thus the identifier attribute provides a unique identifer for the resource. A title attribute captures 


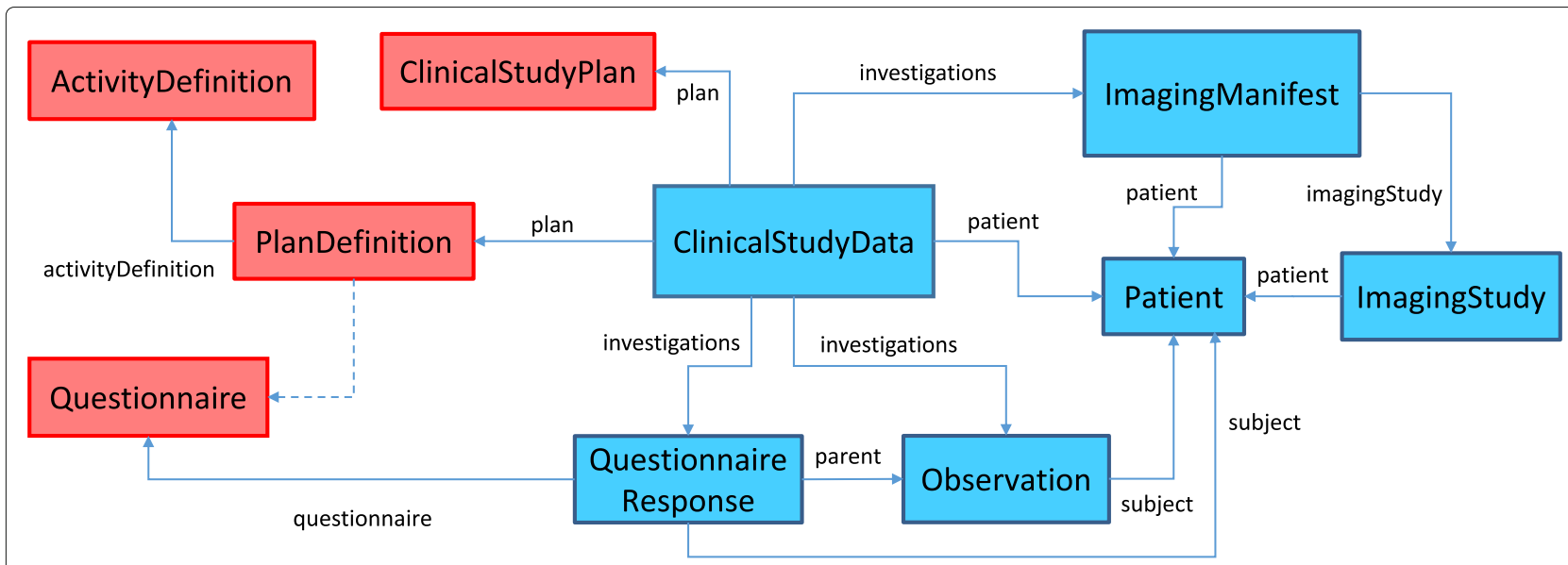

Fig. 4 The clinical research data model in FHIR. Illustrates the metadata (red) and data (blue) resources comprising the clinical data model for describing and capturing the research study natively in FHIR. The study plan can be described using either the ClinicalstudyPlan or $\mathrm{PlanDef}$ inition resource. The latter can be further defined using the ActivityDef inition resource. The Questionnaire resource provides the definition for forms within the study plan. A link to the study plan is contained within the ClinicalstudyData resource. The $\mathrm{Cl}$ inicalstudyData resource encapsulates the clinical data comprising the research study. It facilitates links to the Patient resource, to describe the study participant. It further describes investigations that can be a QuestionnaireResponse or a series of observation or ImagingManifest resources. The ImagingManifest resource further defines an Imagingstudy resource to describe the imaging study being conducted

the title under which the study is publicly known. An officialTitle attribute holds the scientific title of the study. The date of registration of the study is contained in the registrationDate field and the regulatory agency effecting the registration is depicted within the authoringBody field. A mandatory status attribute specifies the current state of the resource. The study sponsors can be described in the sponsor field, which allows a Group resource to be defined. The publiccontact attribute specifies the contact details of the person responsible for general enquiries about the study. An investigator attribute discloses the principal investigator for the study; a person tasked at initiating the study, developing the study protocol and responding to scientific enquiries about the study. A textual description of the aims of the study is provided by the description attribute. The actual or forecasted date of first participant enrolment is recorded in the dateFirstenrolment and the expected total number of participants enrolled is captured in the samplesize attribute.

The desired outcome of the study is captured within the goal attribute. Each goal is further divided into three sub-attributes. The name of the outcome is contained within the outcome attribute. A metric attribute describes the metric or method of measurement used to evaluate the outcome and finally a timepoint attribute records the timepoints of interest in which to achieve the goal.

We then define the activities that constitute the study. In [8], we outlined how the Questionnaire resource is insufficient to capture all activities from clinical studies, especially longitudinal ones. By defining all aspects of actions resulting from clinical studies within activity attributes, we facilitate the definition of both traditional questions and more observational measurements. A scheduled attribute allows the timing of an activity to be defined. We chose an actionResulting attribute to describe the questionnaire developed as part of the activity. We then define a detail attribute to provide a detailed description of sub-activities that will ultimately lead to Observation and ImagingManifest resources in FHIR. This attribute thus provides three sub-attributes to document the category, type and rationale for each sub-activity. We also chose to record the Practitioner or Organisation involved in the activity through the performer attribute and provide a reference to the activity's location using a location attribute. Finally, a note attribute allows any comments relating to the clinical study plan to be recorded.

We have started engaging with the HL7 FHIR-I ${ }^{6}$ [26] and RCRIM ${ }^{7}$ [27] working groups. The FHIR community, however, intends to release, as part of $\mathrm{STU}^{8}{ }^{8}$, a $\mathrm{PlanDefinition}$ resource that captures many of the functionalities of the $\mathrm{ClinicalstudyPlan}$ resource.

\section{PlanDefinition}

PlanDefinition ${ }^{9}$ is a resource proposed by the HL7 community that is at the ballot phase and that they intend to release as part of STU3 in late 2016. We will only comment on the main concepts as this is a draft proposal 


\begin{tabular}{|c|c|c|c|c|c|c|c|}
\hline Structure & UML & XML & & SON & Turtle & R2 Diff & All \\
\hline \multicolumn{8}{|l|}{ Structure } \\
\hline \multicolumn{3}{|c|}{ Name } & Flags & Card. & \multicolumn{2}{|l|}{ Type } & Description \& Constraints \\
\hline \multicolumn{3}{|c|}{ ClinicalstudyPlan } & & & \multicolumn{2}{|c|}{ DomainResource } & Healthcare plan for a clinical study \\
\hline \multicolumn{3}{|c|}{$@$ identifier } & $\Sigma$ & $0 . .^{*}$ & \multicolumn{2}{|l|}{ Identifier } & Primary Ids for this plan \\
\hline \multicolumn{3}{|c|}{ (1) externalidentifier } & $\Sigma$ & 0..* & \multicolumn{2}{|l|}{ Identifier } & External/Secondary Ids relating to the plan \\
\hline \multicolumn{3}{|c|}{ title } & $\Sigma$ & $0 . .1$ & \multicolumn{2}{|l|}{ string } & Public title for the study \\
\hline \multicolumn{3}{|c|}{ officalTitle } & $\Sigma$ & $0 . .1$ & \multicolumn{2}{|l|}{ string } & Scientific title for the study \\
\hline \multicolumn{3}{|c|}{ WregistrationDate } & $\Sigma$ & $0 . .1$ & \multicolumn{2}{|l|}{ dateTime } & Date when the study was first registered \\
\hline \multicolumn{3}{|c|}{ authoringBody } & $\Sigma$ & $0 . .1$ & \multicolumn{2}{|l|}{ string } & Authority that facilitates the registration of the study \\
\hline \multicolumn{3}{|c|}{ status } & $? ! \Sigma$ & $1 . .1$ & \multicolumn{2}{|l|}{ code } & $\begin{array}{l}\text { proposed | draft | active | completed | cancelled } \\
\text { ClinicalStudyPlanStatus (Required) }\end{array}$ \\
\hline ta spon & & & $\Sigma$ & o..* & Reference & Group) & Sponsor(s) for the study \\
\hline E publi & Contact & & & $0 . .1$ & Reference & Practitioner) & The person responsible for general enquiries about the study \\
\hline - $\tau^{x}$ inves & igator & & $\Sigma$ & $0 . .1$ & Reference & Practitioner) & Who's the principal investigator involved in plan? \\
\hline desc & ption & & $\Sigma$ & $0 . .1$ & string & & Textual description of the aims of the study \\
\hline goal & & & $\Sigma$ & 0..* & BackboneE & lement & The desired outcome of the study \\
\hline and or & come & & & $0 . .1$ & string & & The name of the outcome \\
\hline$\infty \mathrm{m}$ & & & $\Sigma$ & $0 . .1$ & Codeablec & oncept & The metric or method of measurement used \\
\hline tin & epoint & & $\Sigma$ & o...* & string & & The timepoint(s) of interest \\
\hline m date & rstEnrolmen & & $\Sigma$ & $0 . .1$ & dateTime & & Date of first participant enrolment \\
\hline sam & esize & & $\Sigma$ & $0 . .1$ & string & & Number of planned participants \\
\hline activ & & & $\Sigma$ & o..* & Backbonet & lement & The activity that occurs as part of the study \\
\hline - ics & eduled $[x]$ & & & $0 . .1$ & & & When activity is to occur \\
\hline$\infty$ & cheduledTin & $\operatorname{ming}$ & & & Timing & & \\
\hline$@$ & scheduledPe & eriod & & & Period & & \\
\hline L & scheduledStr & ring & & & string & & \\
\hline 七 ac & ionResulting & & & $0 . .1$ & Reference & Questionnaire) & The Questionnaire developed \\
\hline de & & & & o..* & BackboneE & lement & Detailed definition of each activity \\
\hline$\infty$ & category & & & $0 . .1$ & Codeablec & ncept & Detail category of activity \\
\hline$\infty$ & code & & & $0 . .1$ & Codeablec & oncept & Detail type of activity \\
\hline L.. & easoncode & & & o..* & Codeablec & ncept & Why activity should be done \\
\hline G & former & & & $0 .{ }^{*}$ & $\begin{array}{l}\text { Reference } \\
\text { Organizati }\end{array}$ & $\begin{array}{l}\text { Practitioner I } \\
\text { n) }\end{array}$ & Who is involved in delivering the activity? \\
\hline .... & ation & & & $0 . .1$ & Reference & Location) & Where was the activity performed? \\
\hline$\ldots$ note & & & & $0 . .1$ & Annotation & & Comments about the plan \\
\hline
\end{tabular}

Fig. 5 The $\mathrm{Cl}$ inicalstudyPlan resource. Describes the elements comprising the $\mathrm{Cl}$ inicalstudyPlan resource. This resource has been generated using the FHIR Build Process [45] based on the FHIR Guide to Designing Resources [46]. The build process builds the resource and generates the webpage that describes the resource, as depicted in this Figure. The table structure is defined in the Resource Definition page [47], which also provides a definition of the flags; '? !' indicates that the element is a modifying element, while ' $\Sigma$ ' indicates that this element is part of the summary set. The activity element allows either the definition of detailed items or a Questionnaire resource to be specified

that is still subject to change at short notice. Unlike the ClinicalStudyPlan, this resource has not been designed to address the planning of clinical research specifically but it is flexible enough to undertake this role. However, similar to the ClinicalstudyPlan resource, it contains attributes to represent the plan's unique identifier, name, status, purpose and contributor. In addition, it defines the version as well as attributes that capture the type of plan defined, the clinical usage foreseen for the plan, a natural language description of the plan, dates of publication and last review, the context of use (coverage) of the plan as well as the topics described.

Central to this resource is the definition of actions (actionDefinition) to occur as part of the plan. Each action has an identifier, a label, a title, a description of the action both in natural language and as
Codeable entities and a link to supporting documentation for the action. Each action further defines a condition for whether as well as some triggers to specify when the action should occur. A description of the activity comprising this action can be further defined within an ActivityDefinition resource.

The proposed ActivityDefinition ${ }^{10}$ resource provides a conceptual description of an action that should be undertaken. Similar to the PlanDefinition resource, it is at the ballot phase and is intended to be released as part of STU3 in late 2016. It contains similar organisational attributes as the PlanDefinition resource. A detailed definition of the activity can be achieved using a CodeableConcept element. A category attribute defines the type of activity undertaken and a timing attribute specifies when the activity should occur. 


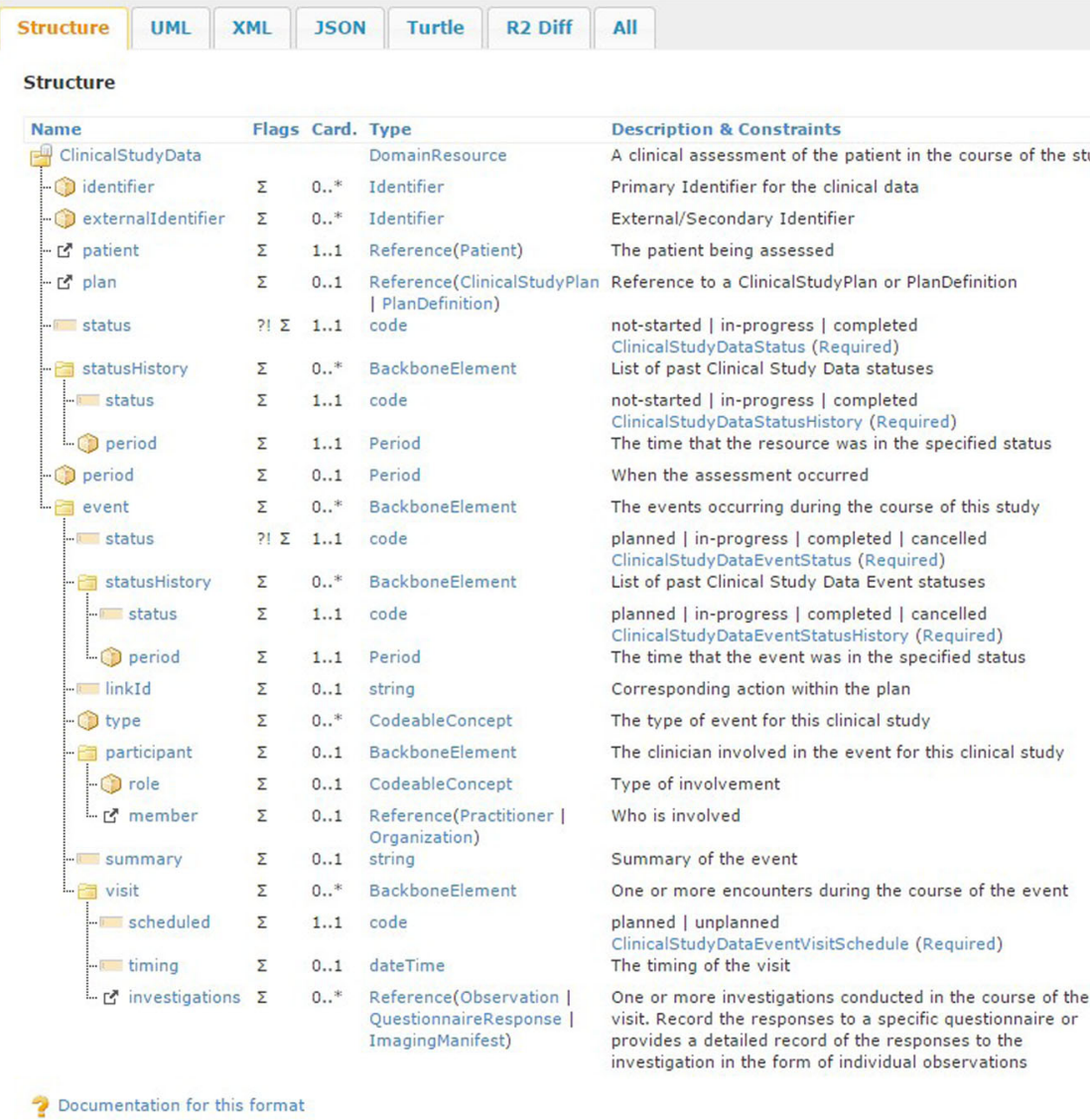

Fig. 6 The $\mathrm{Cl}$ inicalstudyData resource. Describes the elements comprising the $\mathrm{Cl}$ inicalstudyData resource. This resource has also been generated using the FHIR Build Process [45] based on the FHIR Guide to Designing Resources [46]. The table structure is defined in the Resource Definition page [47], which also provides a definition of the flags; '? ' indicates that the element is a modifying element, while ' $\Sigma$ ' indicates that this element is part of the summary set. The event element describes the events occurring throughout the study. An event can be further divided into visits. Each visit defines an invest igation, which can be only one of the following: a QuestionnaireResponse resource or a series of Observation or ImagingManifest resources

\section{ClinicalStudyData}

The ClinicalstudyData resource describes the data captured during the study. An identifier is defined to provide a link to the primary identifiers for the study. For external identifiers, such as a hospital patient id, an externalidentifier attribute is provided. A mandatory patient attribute provides a reference to the patient being assessed. The ClinicalstudyData resource provides a link to either the ClinicalstudyPlan or Plandefinition resource, through the plan attribute, to uniquely identify the study that this clinical data instance represents. A status attribute defines the current state of the resource. We also chose to keep a record of past statuses in a statusHistory attribute that captures the past statuses as well as the time that the event was in the specified state. The time period during which the patient underwent the clinical assessment is depicted in the period attribute.

We then define the event occurring during the course of the study. An event represents the execution of one or more activities during the course of the study to assess the patient. Each event transitions through a number of states and the state is contained within the status attribute. A type attribute describes the type of the event. The clinicians involved in this event are described within the participant attribute, which further defines their role and a reference to the involved member, be it a Practitioner or an Organization. A summary of the event is provided within the summary attribute.

In clinical study parlance, an event can last anything from a few seconds to several months or even years. Consequently, we define a visit attribute to 
describe one or more encounters between the clinician and the patient. Visits can be planned or unplanned and this is defined within the scheduled attribute. The timing of the visit is described within the timing attribute. Finally, we define an investigations attribute to capture one or more clinical investigations during the course of the visit. These take the form of a reference to either a QuestionnaireResponse or a series of Observation or ImagingManifest resources.

\section{Demonstrating the clinical study design with FHIR}

We illustrate the fit of the FHIR data model by discussing a clinical study focussed on cardiovascular episodes. Our focus is to highlight the impact that the addition of contextual information, and their relationships with the data elements, have on the semantic relevance and interpretation of the clinical data. Typically, the output from a clinical study, in ODM XML format, is as depicted below:

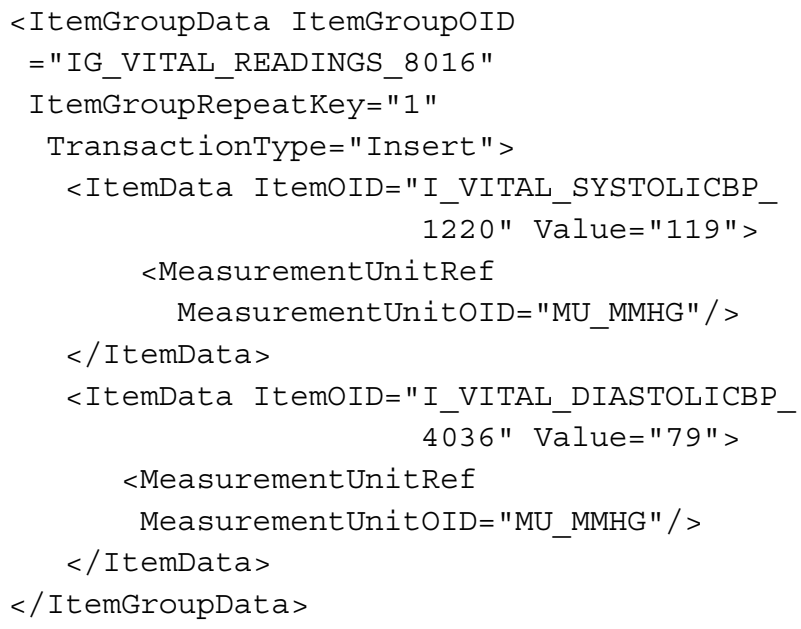

This states that the study participant has a blood pressure of $119 / 79 \mathrm{mmHg}$ but provides no information on how the measurements were obtained. Handler [28] outlines nine factors that may affect the accuracy of blood pressure measurements. To assist the user in making informed decisions about the clinical data, relevant contextual information, such as illustrated below, should be provided with the data. This additional metadata tells us that the readings were taken at 10:00 am by a nurse from the left upper arm and in a sitting position. While it is important to standardise the data and metadata, what is missing is the relationship to the initial blood pressure measurements. When the measurements are presented as a series of unrelated data elements, they cannot reliably be interpreted (Appendix in [5]).

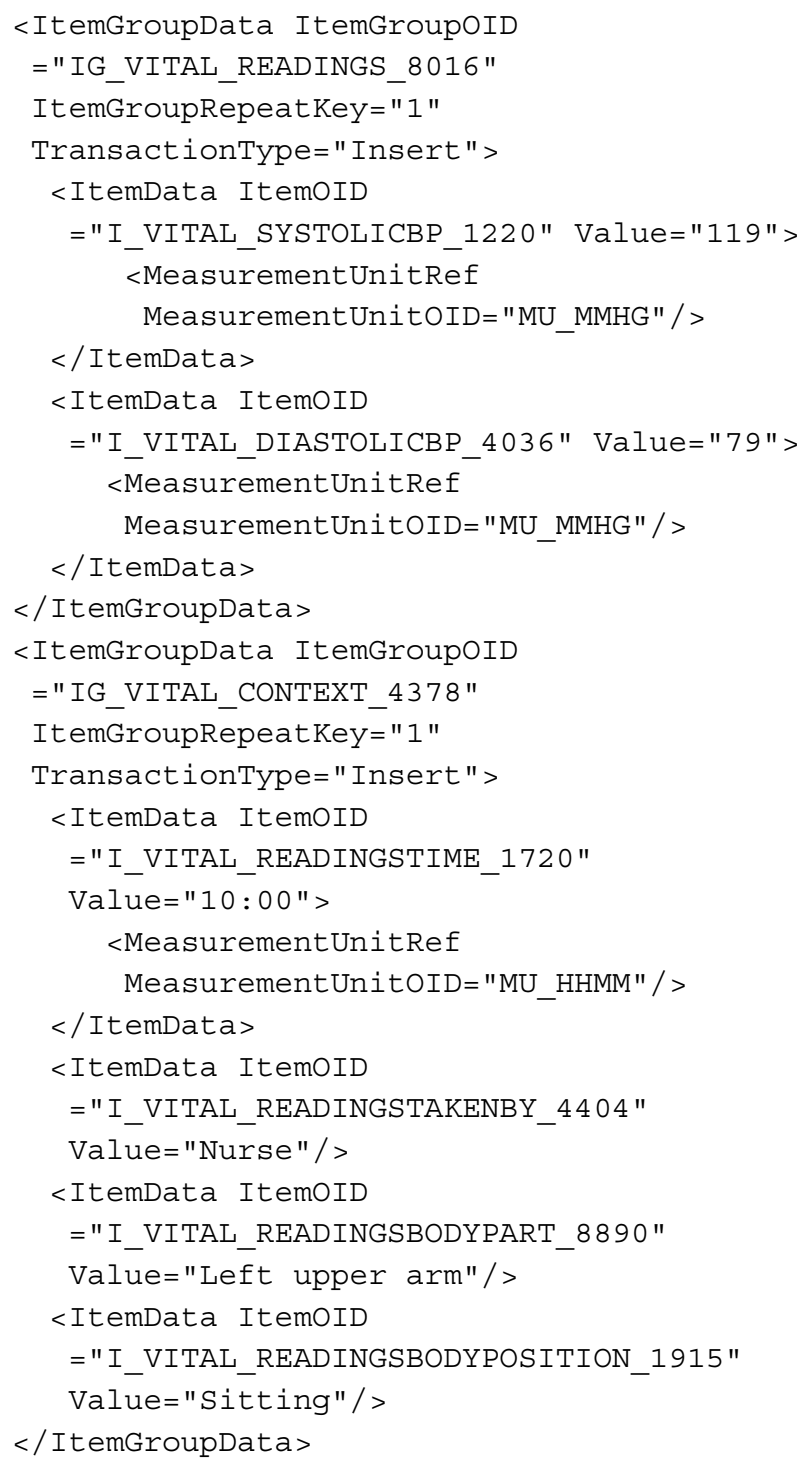

The FHIR framework, in particular resources such as Observation, provides the means to accurately represent the relationships between the data elements so that they can be understood and interpreted more effectively. A representation of the blood pressure above, implemented using the Observation resource, is illustrated in the Additional file 1. In addition to capturing the blood pressure measurements described previously, the Observation resource provides a reference to the Patient resource to identify the study participant and to the Practitioner resource to identify the clinician performing the blood pressure measurement. More importantly, it natively encapsulates the contextual information, such as the body part and body position, as well as the ability to interpret the measurements.

By taking advantage of resources that encapsulates rich interrelated clinical data, as demonstrated by the Observation resource, the ClinicalstudyData 
resource facilitates the definition of the entire research study, in terms of the subjects enrolled; the clinical data associated with these subjects and the experiments undertaken. In addition, it provides a framework, through the plan element, for the study plan to be associated with the clinical data. An implementation of the ClinicalStudyData and ClinicalStudyPlan resources is provided at http:// healthinet.it.csiro.au/net/jbs/.

\section{Discussion}

The implementations of the ClinicalstudyPlan and ClinicalstudyData resources demonstrate the fit of the FHIR standard in capturing and managing clinical data from research studies. The pertinence of this finding is that the clinical data no longer need to be transformed from an arbitrary standard into FHIR resources, thus reducing the risk of introducing errors and losing fidelity. The proposed models achieve semantic interoperability by defining a set of common elements for describing the actions performed on the data as well as defining common elements for describing the data and its context through the use of controlled terminologies and ontologies. This, then allows the resources to be shared and processed across systems. The FHIR resources provide the means to navigate and access the clinical data at numerous levels with the addition of several dimensions at the patient, event, activity and data item level, thereby negating the limitations of the monolithic and rigid hierarchy of the ODM data model.

The World Health Organisation (WHO) has released a list of twenty mandatory items for the definition of a study protocol [29] so that the given trial can be considered fully registered. We present, in Table 1, a listing of the twenty items alongside the attributes from the ClinicalstudyPlan and PlanDefinition resources. We have not provided a study type as the ClinicalStudyPlan inherently suggests a clinical study. We have also chosen not to explicitly define the source of monetary funds and countries of recruitment as these are primarily associated with clinical trials. However, it is our support for eligibility criteria that is particularly inadequate. In our defence, our focus here has been the definition of an alternative structural representation to CDISC ODM for clinical study design. Furthermore, we regard the formulation of an effectual eligibility criteria as non-trivial and one that we deemed out of scope for this paper. We intend to engage with the HL7 community to embed computable study protocol criteria within our resource as adequate representation of the study protocol is very useful and important [10]. Previous attempts, such as the CDISC Protocol Representational Model (PRM), have had limited adoption by the clinical study community [10]. (PRM [30] is a UML ${ }^{11}$-based standard that developed a set of
Table 1 Listing of the 20 WHO items for clinical study protocol

\begin{tabular}{|c|c|c|}
\hline $\begin{array}{l}\text { WHO trial registration } \\
\text { data set }\end{array}$ & PlanDefinition & ClinicalStudyPlan \\
\hline $\begin{array}{l}\text { 1. Primary registry and } \\
\text { trial identifying number }\end{array}$ & Identifier & Identifier \\
\hline $\begin{array}{l}\text { 2. Date of registration in } \\
\text { primary registry }\end{array}$ & & RegistrationDate \\
\hline $\begin{array}{l}\text { 3. Secondary identifying } \\
\text { numbers }\end{array}$ & Identifier & Externalldentifier \\
\hline \multicolumn{3}{|l|}{$\begin{array}{l}\text { 4. Source(s) of monetary or } \\
\text { material support }\end{array}$} \\
\hline 5. Primary sponsor & & Sponsor \\
\hline 6. Secondary sponsor(s) & & Sponsor \\
\hline $\begin{array}{l}\text { 7. Contact for public } \\
\text { queries }\end{array}$ & Publisher & PublicContact \\
\hline $\begin{array}{l}\text { 8. Contact for scientific } \\
\text { queries }\end{array}$ & & Investigator \\
\hline 9. Public title & Title & Title \\
\hline 10. Scientific title & & OfficialTitle \\
\hline \multicolumn{3}{|l|}{$\begin{array}{l}\text { 11. Countries of } \\
\text { recruitment }\end{array}$} \\
\hline $\begin{array}{l}\text { 12. Health condition(s) or } \\
\text { problem(s) studied }\end{array}$ & Purpose* & Description \\
\hline 13. Intervention(s) & ActionDefinition & Activity \\
\hline \multicolumn{3}{|l|}{$\begin{array}{l}\text { 14. Key inclusion and } \\
\text { exclusion criteria }\end{array}$} \\
\hline 15. Study type & Type $^{*}$ & * \\
\hline 16. Date of first enrolment & & DateFirstEnrolment \\
\hline 17. Target sample size & & SampleSize \\
\hline 18. Recruitment status & Status & Status \\
\hline 19. Primary outcome(s) & Coverage $^{*}$ & Goal \\
\hline $\begin{array}{l}\text { 20. Key secondary } \\
\text { outcomes }\end{array}$ & Coverage $^{*}$ & Goal \\
\hline
\end{tabular}

standardised protocol concepts that was intended to be used alongside the other CDISC and HL7 standards.)

The appeal in definining a visit as part of an event in the ClinicalstudyData resource is to more accurately describe protracted events within multimodal longitudinal clinical studies. It is often useful, in the case of lengthy events, to be able to define a sub-event and subsequently record the study participants' attendance to the sub-event. Consequently, the outcome of those visits can be represented as a QuestionnaireResponse, ImagingManifest or an Observation through the investigations attribute. The QuestionnaireResponse, ImagingManifest and Observation resources suit different types of clinical studies [8]. The pertinence of the Observation resource is the ability to store important contextual information alongside the clinical data, the ability to interpret 
the observation in the context of a controlled vocabulary or ontology and the ability to provide some justification as to the absence of a measurement [8].

While the PlanDefinition resource can be used to describe the study plan, it still has some inadequacies to overcome. As the PlanDefinition resource has not been specifically designed to address the planning of clinical research, it logically has to be more generic. Consequently, it is unclear how the PlanDefinition resource relates to the FHIR resources designed to capture the clinical data that it defines. Furthermore, it is also unclear what mechanism is envisaged to ensure that the data capture resources conform to the plan definition. Moreover, as the resource has not been designed for a clinical research domain, the PlanDefinition resource also lacks the necessary mechanisms to fully define the study protocol. In particular, it does not offer the option of recording the date of registration, the sponsor $(s)$, the date of enrolment, expected sample size, study type and study outcome ( $\mathrm{s})$. More importantly, in our view, is the lack of support for machine-processable inclusion and exclusion criteria to be embedded within the PlanDefinition resource. While the PlanDefinition defines a trigger and a condition element, these relate to the execution of the PlanDefinition resource and do not constitute the definition of the conditions addressing the eligibility of the participants to participate in the study. We advocate for the eligibility criteria to be designed in a manner to influence and advance the study design and form generation as outlined in [3] in their five phases of clinical research data lifecycle.

While the ClinicalStudyPlan and PlanDefinition resources are structurally similar, there are subtle differences between them. It is unclear how the Questionnaire resource (indicated by dotted lines in Fig. 4) fits within the PlanDefinition resource. This may, in our view, restrict its ability to be used for anthropological studies or surveys.

\section{Related work}

Prior to FHIR, several information models have been proposed to standardise the representation of clinical information. The Clinical Element Model (CEM) is an information model designed to provide a consistent architecture for representing clinical information in EHR systems [31]. The ISO 13606 standard is an international standard published by ISO that specifies the information models and vocabularies needed for the interoperability of EHR systems [32]. Both models aim to address the issue of semantic interoperability by standardising the data, metadata and their relationships similar to our approach.

Numerous research have centred around the CDISC models recently. Dugas [25] describes two tools to convert forms between the CDISC ODM and HL7 $\mathrm{CDA}^{12}$ formats to facilitate the sharing of electronic health records (EHRs) and clinical data to address the problem of redundant documentation in both systems. His findings reflect our position that the conversion process is lossy because the CDISC and HL7 models serve different purposes and hence have different properties. Similarly, the SALUS project $[33,34]$ is a former attempt to adapt CDISC standards to build a semantic framework to improve the interoperability between clinical research and clinical care domains. More specifically, it looks at combining the strengths of CRFs with those of EHRs to address adverse drug reactions. We envisage our proposed FHIR clinical study model to facilitate the incorporation of existing EHR data to augment the capabilities of retrospective observational studies similar to their approach. Jiang et al. [35] have developed and evaluated a Semantic Web-based approach for the generation of domain-specific templates from the integration of the BRIDG model and the ISO 21090 data types, to support clinical study metadata standards development. Vadakin and Hinkson [36] discuss the CDISC PRM and outline its importance in supporting research study design, registration, tracking and in providing a single-source of protocol content electronically. They stress that typical protocol document is not useful for information management and re-use. PRM standardises the protocol content into a structured document that is easier to understand and to exchange, in machinereadable format, across systems [36]. We are mindful of their findings in order to address the issue of the protocol definition within our research data model.

A topical area of research has been the standardisation and structuring of clinical forms. Abler et al. [18] discuss the need for a language for forms that can effectively record the logical relationships between questions or sets of questions asked in the forms. Richesson and Nadkarni [11] provide a review of the electronic data capture standards landscape and discuss their current limitations. Bruland et al. [9] discuss the standardisation of CRFs to achieve interoperability in clinical research. They outline the difficulties of promoting the standardising and structured representation of forms in the context of data exchange and propose a mapping model between the National Cancer Institute forms and CDISC ODM files semantically annotated using the Alias element. As stated in [8], the tendency would be to organise the forms within a Questionnaire resource in FHIR. However, this understates the nature of the information captured and the choice of a QuestionnaireResource, observation and ImagingManifest resource ensures the optimal capture of the information.

The Linked Clinical Data Cube (LCDC) $[6,7,19]$ describes a semantic web approach to investigate the association of the semantic statistics vocabularies with 
clinical data exchange standards and demonstrate their fit in achieving the semantic enrichment of clinical study data with a view to fulfilling semantic interoperability. The LCDC defines a set of modularised data cubes that helps manage the multi-dimensional and multidisciplinary nature of clinical data. It requires mapping to the RDF Data Cube [37] and DDI ${ }^{13}$-RDF Discovery [38] vocabularies to organise the data and links to domain ontologies to semantically enrich it. The LCDC represents the precursor to our data model in FHIR. The HL7 working group on Semantic Interoperability [39] has initiated work on translating the XML and JSON version of FHIR into FHIR RDF. Once completed, this should allow the integration of the FHIR data model with the semantic statistics vocabularies.

\section{Future work}

We intend to engage with the FHIR community to address the full support for the definition of eligibility criteria within the FHIR resources. There is a need for the current text-based criteria to be formalised and provided in machine-readable format to facilitate computerised determination of eligibility [10]. Machine-processable definition of eligibility criteria will not only mould the study design but can influence the patients' recruitment process as outlined in [40].

The FHIR specification provides the functionality, through the FHIR mapping language [41], to transform clinical data from one model to another. We intend to take advantage of this functionality to map the FHIR clinical data model back to the CDISC standards. As outlined earlier, the regulatory bodies favour the use of the CDISC standards for the reporting of clinical studies [5]. By using FHIR to model the clinical study data, we capture the contextual information, and fulfil the requirements of the FDA by retrofitting the clinical data to the CDISC models.

We also aim to support the formulation of temporal constraints to assist in the scheduling of activities as outlined in [42], which describes a knowledge-based approach to specifying and monitoring temporal constraints in relational databases.

\section{Conclusion}

This paper has presented a proposal for a clinical study architecture to support the semantic interoperability of clinical data using the FHIR resources. We have shown how the clinical research community is likely to benefit from the adoption of FHIR resources to capture and manage clinical study data. In this regard, we have outlined a method to link clinical data from the XML-based CDISC ODM model to a selective group of FHIR resources. While we have revealed a fit between the ODM model and the
FHIR resources, we do not regard this as a long term solution. First, owing to the evolving nature of the FHIR specifications, this mapping is likely to change at a whim. Second, it is preferable to avoid data transformations but for data to be captured directly at the source. We have thus proposed two FHIR models, a ClinicalstudyPlan and a ClinicalstudyData resource, and shown that they can natively manage clinical data. We have compared our work to the proposed HL7 PlanDefinition FHIR resource and discussed their suitability in adequately representing the research study protocol definition. We have demonstrated, with the help of a working example, the fit of our clinical data model in interpreting clinical research data. Our work has built the foundations to not only facilitating the syntactic but also semantic interoperability of clinical research data.

\section{Endnotes}

${ }^{1}$ Extensible Markup Language [43]

${ }^{2}$ Health Level Seven

${ }^{3}$ Based on the 'Standard for Trial Use 3' September 2016 version

${ }^{4}$ Systematized Nomenclature of Medicine Clinical Terminology

${ }^{5}$ Logical Observation Identifiers Names and Codes

${ }^{6}$ FHIR-Infrastructure

${ }^{7}$ Regulated Clinical Research Information Management

${ }^{8}$ Standard for Trial Use 3

${ }^{9}$ This resource can be found at http://hl7.org/fhir/ 2016Sep/plandefinition.html

${ }^{10}$ This resource can be found at http://hl7.org/fhir/ 2016Sep/activitydefinition.html

${ }^{11}$ Unified Modeling Language

${ }^{12}$ Clinical Document Architecture

${ }^{13}$ Data Documentation Initiative

\section{Additional file}

Additional file 1: The file observation_example.json lists the Observation resource as described in the Demonstrating the clinical study design with FHIR section in json format. (JSON $2 \mathrm{~kb}$ )

\section{Abbreviations}

CDA: Clinical document architecture; CDASH: Clinical data acquisition standards harmonization; CDISC: Clinical data interchange standards consortium; CEM: Clinical element model; DSTU2: Draft standard for trial use 2; FHIR: Fast Healthcare interoperable resources; FHIR-I: FHIR-Infrastructure; HL7: Health level seven; ISO: International organization for standardization; LCDC: Linked clinical data cube; LOINC: Logical observation identifiers names and codes; ODM: Operational data model; RCRIM: Regulated clinical research information management; SNOMED CT: Systematized nomenclature of medicine clinical terminology; STU3: Standard for trial use 3; XML: Extensible markup language 


\section{Acknowledgements}

The authors would like to thank Drs Marlien Varnfield and Parnesh Raniga for reviewing the paper.

An initial version of this paper has been published on Researchgate at [44].

\section{Funding}

This research has not been funded from an external grant.

\section{Availability of data and materials}

The mapping of the clinical data from CDISC ODM to FHIR can be accessed at: http://healthinet.it.csiro.au/net/jbs/odmFhir.

The web resources and implementation of the new FHIR resources have been included at: http://healthinet.it.csiro.au/net/fhir/.

A working example showcasing the two FHIR resources can be found at: http://healthinet.it.csiro.au/net/jbs/implementation.

\section{Authors' contributions}

$\mathrm{HL}$ designed the FHIR resources that $\mathrm{AM}$ and $\mathrm{ML}$ reviewed. $\mathrm{HL}$ implemented the FHIR resources and part of the clinical study examples and drafted the manuscript. AM implemented part of the clinical study examples. AM and ML reviewed the manuscript. All authors read and approved the final manuscript.

\section{Ethics approval and consent to participate}

Not applicable.

\section{Consent for publication}

Not applicable.

\section{Competing interests}

The authors declare that they have no competing interests.

\section{Publisher's Note}

Springer Nature remains neutral with regard to jurisdictional claims in published maps and institutional affiliations.

Received: 2 June 2016 Accepted: 3 September 2017

Published online: 19 September 2017

\section{References}

1. van Valkenhoef G, Tervonen T, de Brock B, Hillege H. Deficiencies in the transfer and availability of clinical trials evidence: a review of existing systems and standards. BMC Med Inform Decis Making. 2012;12(1):95.

2. Hsu W, Gonzalez NR, Chien A, Villablanca JP, Pajukanta P, Viñuela F, Bui AA. An integrated, ontology-driven approach to constructing observational databases for research. J Biomed Inform. 2015;55:132-42.

3. Hume S, Aerts J, Sarnikar S, Huser V. Current applications and future directions for the CDISC Operational Data Model standard: A methodological review. J Biomed Inform. 2016;60:352-62.

4. CDISC: Specification for the Operational Data Model (ODM). 2006. http:// www.cdisc.org/odm-v1-3-2. Accessed 26 July 2017.

5. FDA: Study data technical conformance guide. 2016. http://www.fda.gov/ downloads/Forlndustry/DataStandards/StudyDataStandards/ UCM384744.pdf. Accessed 26 July 2017.

6. Lefort L, Leroux H. Design and generation of linked clinical data cubes. In: Proceedings of 1st International Workshop on Semantic Statistics (SemStats 2013). Sydney: CEUR Workshop Proceedings; 2013.

7. Leroux H, Lefort L. Semantic enrichment of longitudinal clinical study data using the CDISC standards and the semantic statistics vocabularies. J Biomed Semantics. 2015;6(1):16.

8. Leroux H, Metke-Jimenez A, Lawley MJ. ODM on FHIR: Towards achieving semantic interoperability of clinical study data. In: Proceedings of Semantic Web Applications and Tools for the Life Sciences (SWAT4LS). Cambridge: CEUR Workshop Proceedings; 2015.

9. Bruland P, Breil B, Fritz F, Dugas M. Interoperability in clinical research: from metadata registries to semantically annotated CDISC ODM. Stud Health Technol Inform. 2011;180:564-8.

10. Huser V, Sastry C, Breymaier M, Idriss A, Cimino JJ. Standardizing data exchange for clinical research protocols and case report forms: An assessment of the suitability of the Clinical Data Interchange Standards Consortium (CDISC) Operational Data Model (ODM). J Biomed Inform. 2015;57:88-99.
11. Richesson RL, Nadkarni P. Data standards for clinical research data collection forms: current status and challenges. J Am Med Inform Assoc. 2011;18(3):341-6. http://jamia.oxfordjournals.org/content/18/3/341.full. pdf. Accessed 26 July 2017.

12. Leroux H, McBride S, Gibson S. On selecting a clinical trial management system for large scale, multi-centre, multi-modal clinical research study. Stud Health Technol Inform. 2011;168:89-95.

13. Leroux H, McBride S, Lefort L, Kemp M, Gibson S. A method for the semantic enrichment of clinical trial data. Stud Health Technol Inform. 2012;178:111-6.

14. ISO: Semantic Interoperability of health information. http://www.springer. com/cda/content/document/cda_downloaddocument/ 9783642225017-c7.pdf. Accessed 26 July 2017.

15. Macía I. Towards a semantic interoperability environment. In: e-Health Networking, Applications and Services (Healthcom), 2014 IEEE 16th International Conference On. IEEE; 2014. p. 543-8. doi:10.1109/HealthCom.2014.7001900.

16. CDISC: Clinical Data Acquisition Standards Harmonization (CDASH) v1.1 2006. http://www.cdisc.org/cdash/. Accessed 26 July 2017.

17. BRIDG: The Biomedical Research Integrated Domain Group (BRIDG) Model. 2016. http://www.bridgmodel.org/. Accessed 26 July 2017.

18. Abler D, Crichton C, Welch J, Davies J, Harris S. Models for forms. In: Proceedings of the Compilation of the Co-located Workshops on DSM'11, TMC'11, AGERE!'11, AOOPES'11, NEAT'11, \& VMIL'11. ACM; 2011. p. 13-18. doi:10.1145/2095050.2095054.

19. Leroux H, Lefort L. Using CDISC ODM and the RDF Data Cube for the semantic enrichment of longitudinal clinical trial data. In: Proceedings of Semantic Web Applications and Tools for the Life Sciences (SWAT4LS). Paris: CEUR Workshop Proceedings; 2012.

20. HL7: Fast Healthcare Interoperable Resources. 2015. http://hl7.org/fhir/ overview.html. Accessed 26 July 2017.

21. HL7: Argonaut project. 2016. http://argonautwiki.hl7.org/. Accessed 26 July 2017.

22. ADHA: National Clinical Terminology Service. 2016. http://www. healthterminologies.gov.au/. Accessed 26 July 2017.

23. HL7: Organizations interested in FHIR. 2016. https://www.cdisc.org/ standards/domain-information-module/bridg. Accessed 26 July 2017.

24. Kubick W. CR 3.0 - A Manifesto for The Next Generation of Clinical Research Data Standards. 2016. https://waynekubick.com/2016/08/30/cr3-0-a-manifesto-for-the-next-generation-of-clinical-research-datastandards/. Accessed 26 July 2017.

25. Dugas M. ODM2CDA and CDA2ODM: Tools to convert documentation forms between EDC and EHR systems. BMC Med Inform Decis Making. 2015;15(1):40.

26. HL7: FHIR-I. 2016. http://www.hl7.org/Special/committees/fiwg/index. cfm. Accessed 26 July 2017.

27. HL7: RCRIM. 2016. http://www.hl7.org/Special/Committees/rcrim/index. cfm. Accessed 26 July 2017.

28. Handler J. The importance of accurate blood pressure measurement. Permanente J. 2009;13(3):51-4.

29. WHO: WHO Trial Registration Data Set (Version 1.2.1). 2016. http://www. who.int/ictrp/network/trds/en/. Accessed 26 July 2017.

30. CDISC: Protocol Representation Model v1. 2010. http://www.cdisc.org/ protocol. Accessed 26 July 2017.

31. Tao C, Jiang G, Oniki TA, Freimuth RR, Zhu Q, Sharma D, Pathak J, Huff SM, Chute CG. A semantic-web oriented representation of the clinical element model for secondary use of electronic health records data. J Am Med Inform Assoc. 2013;20(3):554-62. http://jamia.oxfordjournals.org/ content/20/3/554.full.pdf. Accessed 26 July 2017.

32. Santos MR, Bax MP, Kalra D. Building a logical EHR architecture based on ISO 13606 standard and semantic web technologies. Stud Health Technol Inform. 2009;160(Pt 1):161-5.

33. Declerck G, Hussain S, Daniel C, Yuksel M, Laleci GB, Twagirumukiza M, Jaulent MC. Bridging data models and terminologies to support adverse drug event reporting using EHR data. Methods Inf Med. 2015;54(1): 24-31.

34. Laleci GB, Yuksel M, Dogac A. Providing semantic interoperability between clinical care and clinical research domains. Biomed Health Inform IEEE J. 2013;17(2):356-69.

35. Jiang G, Evans J, Endle CM, Solbrig HR, Chute CG. Using semantic web technologies for the generation of domain-specific templates to support 
clinical study metadata standards. J Biomed Semant. 2016;7(1):1-10. doi:10.1186/s13326-016-0053-5.

36. Vadakin A, Hinkson B. Organizing and Accelerating the Clinical Research Process from the Beginning: The CDISC Protocol Representation Model and Toolkit. 2012. http://www.cdisc.org/system/files/all/standard_ category/application/pdf/prm_document__april_2012fin.pdf. Accessed 26 July 2017.

37. Cyganiak R, Reynolds D, Tennison J. The RDF Data Cube Vocabulary. World Wide Web Consortium. 2013. http://www.w3.org/TR/2013/PRvocab-data-cube-20131217/. Accessed 26 July 2017.

38. Bosch T, Cyganiak R, Wackerow J, Zapilko B. Leveraging the DDI Model for Linked Statistical Data in the Social, Behavioural, and Economic Sciences. 2012. In: International Conference on Dublin Core and Metadata Applications; 2012. p. 46-55. http://dcpapers.dublincore.org/pubs/ article/viewFile/3654/1877.

39. HL7: RDF for Semantic Interoperability. 2016. http://wiki.hl7.org/index. php?title=RDF_for_Semantic_Interoperability. Accessed 26 July 2017.

40. Dameron O, Besana P, Zekri O, Bourdé A, Burgun A, Cuggia M, et al. OWL model of clinical trial eligibility criteria compatible with partially-known information. J Biomed Semant. 2013;4:17.

41. HL7: FHIR Mapping Language. 2016. http://build.fhir.org/mappinglanguage.html. Accessed 26 July 2017.

42. O'Connor MJ, Shankar RD, Parrish DB, Das AK. Knowledge-data integration for temporal reasoning in a clinical trial system. Int J Med Inform. 2009;78:77-85.

43. W3C: Extensible Markup Language. 2016. https://www.w3.org/XML/. Accessed 26 July 2017.

44. Leroux H, Metke-Jimenez A, Lawley MJ. Achieving semantic interoperability of clinical study data with FHIR. 2016. https://www. researchgate.net/publication/303839490_Achieving_semantic interoperability_of_clinical_study_data_with_FHIR. Accessed 26 July 2017.

45. HL7: FHIR Build Process. 2016. http://wiki.hl7.org/index.php?title=FHIR_ Build_Process. Accessed 26 July 2017.

46. HL7: FHIR Guide to Designing Resources. 2016. http://wiki.hl7.org/index. php?title=FHIR_Guide_to_Designing_Resources. Accessed 26 July 2017.

47. HL7: Resource Formats. 2016. http://build.fhir.org/formats.html. Accessed 26 July 2017.

\section{Submit your next manuscript to BioMed Central and we will help you at every step:}

- We accept pre-submission inquiries

- Our selector tool helps you to find the most relevant journal

- We provide round the clock customer support

- Convenient online submission

- Thorough peer review

- Inclusion in PubMed and all major indexing services

- Maximum visibility for your research

Submit your manuscript at www.biomedcentral.com/submit 